De quelques enjeux et usages historiques du Français fondamental

\title{
L'enseignement du vocabulaire en Espagne avant et après le Français fondamental
}

Maria Eugenia Fernández Fraile

\section{(2) OpenEdition \\ Journals}

Édition électronique

URL : https://journals.openedition.org/dhfles/1212

DOI : $10.4000 /$ dhfles. 1212

ISSN : 2221-4038

Éditeur

Société Internationale pour l'Histoire du Français Langue Étrangère ou Seconde

Édition imprimée

Date de publication : 1 janvier 2006

Pagination : 195-214

ISSN : 0992-7654

Référence électronique

Maria Eugenia Fernández Fraile, «L'enseignement du vocabulaire en Espagne avant et après le Français fondamental », Documents pour l'histoire du français langue étrangère ou seconde [En ligne], 36 | 2006, mis en ligne le 20 novembre 2014, consulté le 27 mai 2021. URL : http:// journals.openedition.org/dhfles/1212 ; DOI : https://doi.org/10.4000/dhfles.1212

Ce document a été généré automatiquement le 27 mai 2021.

(c) SIHFLES 


\title{
L'enseignement du vocabulaire en Espagne avant et après le Français fondamental
}

\author{
Maria Eugenia Fernández Fraile
}

\section{Le contexte}

1 L'Espagne est le pays où le Français fondamental (FF) a eu l'une des plus larges répercussions. Dans le jugement que $\mathrm{P}$. Rivenc porte sur le $\mathrm{FF}$, vingt-cinq ans après sa parution (1979: 15), il souligne le caractère de " monument historique » du FF en affirmant qu'il appartient au "patrimoine culturel d'une certaine génération de professeurs de français. " Et il ajoute que son relief est tel que, « dans certains pays, comme l'Espagne, il constitue très officiellement la base de référence des contenus linguistiques du programme des deux premières années de français. » $(1979: 15)$ C'est ce que nous allons essayer de montrer. Pour comprendre l'impact énorme que le FF a eu en Espagne, nous devons auparavant présenter de façon très brève quelle était la situation en Espagne, concernant la fixation des contenus lexicaux dans les programmes et les manuels, ainsi que l'enseignement du lexique. Ceci fait, nous serons mieux en mesure d'apprécier le bouleversement qui s'est produit.

2 Pour comprendre comment se pose la question du vocabulaire dans l'enseignement $d u$ français langue étrangère (FLE) en Espagne, il faut partir du Cuestionario (ou Programme officiel) de Lenguas Vivas de 1927. Ce Cuestionario déborde le cadre habituel des Programmes antérieurs, et rejoint le format des Instructions sur les langues vivantes qui étaient publiées à l'époque en France (celle de 1925, par exemple). Il établit bien sûr les contenus de phonétique, de grammaire et de lexique, en fixant leur progression; il mène aussi une réflexion sur l'état de l'enseignement des LE en Espagne, il rentre dans le domaine de la méthodologie, et, sur un ton conciliant mais dirigiste, il souligne fortement le rôle de la conversation, et conseille l'induction comme système de compréhension des règles de grammaire, etc. 
3 Pour la première fois en Espagne, une réflexion linguistique est proposée par les Instructions officielles. À la question : "Quelle langue doit-on enseigner ? », le Cuestionario répond : « la langue courante ou usuelle, l'ensemble des faits linguistiques qui expriment les manifestations permanentes de la vie dans l'ordre physique, intellectuel et social $»^{1}$. La disposition, ou la progression de ce vocabulaire, reprend l'idée de la " méthode cyclique », c'est-à-dire qu'à un vocabulaire « fondamental » (sic) appris initialement, à savoir les mots proches de la réalité de l'élève et de ses « centres d'intérêt » (la salle de classe, la famille, la maison, le village), on ajoute dans les cours suivants « de nouveaux mots [appartenant] aux vocabulaires technique et littéraire ». En tout cas, l'enseignement de ce vocabulaire ne doit pas être fait sous forme de « cours méthodiques sur les matières concernées ", mais sous " forme de conversations ». Le contenu culturel fixé par le Cuestionario de 1927 établit les domaines lexicaux ou " temas » qui serviront de base à la fixation du vocabulaire que les élèves devront apprendre : l'organisation de l'Église catholique, le théâtre, le commerce, la navigation maritime, l'État, l'administration de la justice, l'organisation de l'enseignement, la famille, l'industrie alimentaire, le vêtement, le bâtiment et l'architecture, l'histoire en général, l'astronomie, la météorologie, la géographie, la botanique...

4 La porte était ouverte à une nouvelle façon de "penser » le vocabulaire. J. Lopez Barrera, agrégé (catedrático) du lycée de Santander, compose un ouvrage, dès 1927, où il dresse un catalogue des termes usuels pour la première et seconde année de français, en suivant les consignes du Cuestionario. Il reprend précisément dans son Préambule une longue citation du Cuestionario, qui indique que la " langue à enseigner est la langue courante ou usuelle ». Les mots y sont regroupés aussi par centres d'intérêt ou " temas » : la salle de classe, les aliments, les vêtements, la maison, la campagne, le petit village, le temps, l'espace, etc. Chaque centre d'intérêt possède une soixantaine de termes. On obtient un total de 1800 mots pour la première année, et de 1400 pour la deuxième année. Le vocabulaire usuel comprend ainsi pour l'auteur, des termes tels que - pour ne prendre que l'exemple des aliments - : les huîtres, le gratin, le gibier, le gigot, une côtelette, la volaille, les entremets, un beignet, une gaufre, un pâté farci (un pastel relleno ?)..., ce qui laisse penser qu'on mangeait bien à l'époque ! Il accompagne les termes de leur traduction française, et fait des remarques sur la traduction de certains termes (doubles acceptions), sur certaines expressions (carta certificada : lettre recommandée, etc.), sur le genre des mots, etc.

5 La destinée de ce Cuestionario fut de courte durée. Il fut remplacé par un nouveau Cuestonario en 1934 (21-X-1934), complémentaire d'un nouveau Plan d'Études (29VIII-1934), qui élimine la progression " lexico-thématique » et la remplace par une volonté plus marquée visant la pratique orale de la langue. Le gouvernement de la République, radical-socialiste, veut marquer ses distances face au Plan d'Études antérieur et propose une méthode mixte : au cours des deux premières années, dont l'objectif consiste à " apprendre de façon pratique la langue ", on recommande la méthode directe, où « le professeur utilisera continuellement la langue française depuis les premiers instants, en évitant les sèches listes de vocabulaire et en insérant celui-ci dans des phrases simples. Il aura recours à l'exhibition d'objets et de tableaux muraux ». Dans les deux années suivantes, où l'objectif consiste à faire " connaître la civilisation française dans ses lignes générales : littérature et art, à travers les œuvres des grands écrivains ", l'accès aux textes littéraires fait basculer les pratiques de classe vers un enseignement traditionnel (cours de grammaire, lectures, explications du 
professeur, questions sur les textes, commentaires, exercices de grammaire, " compositions ", etc.).

6 L'Espagne paraissait s'aligner sur ce qui se faisait en Europe à l'époque : sauf que le dénouement de la guerre civile va tout faire revenir en arrière, d'une cinquantaine d'années dans notre domaine. Pour une information plus poussée sur l'enseignement du FLE pendant cette période, je renvoie à deux thèses soutenues (2003). La première, qui concerne les années 1938-1970, a été soutenue par B. Urbano à l'université de Grenade ; la deuxième, qui concerne les années 1936-1970, est due à J. Morales Gil à l'université de Huelva (voir références bibliographiques). Je vais me limiter à dresser un bref état des lieux sur la question du lexique et sur les tendances qui se dessinent.

7 Les programmes officiels pour les langues étrangères qui émanent du Plan d'Études de 1938 sont conçus dans l'objectif global des législateurs de «transformer la société» (Utande Igualada 1975: 75), et de " transformer les mentalités de la Nouvelle-Espagne, en expulsant de nos milieux intellectuels : le manque de [...] formation doctrinale et morale, le mimétisme philo-étranger, la russophilie, l'efféminement, la déshumanisation de la littérature et de l'art. " (Ley reguladora de los estudios de Bachillerato, 20-IX-1938, Bulletin officiel (BOE) : 1387) Pour ce faire, «la formation classique et humaniste doit être accompagnée d'un contenu éminemment catholique et patriotique » (BOE $1938:$ 1386). Certains paragraphes de cette loi appellent au retour à 1' " Être authentique de l'Espagne ", qui a été forgé par les études classiques et humanistes de notre XVIe siècle »! (ibid. : 1386). Il s'agit bien sûr des valeurs consacrées par la Contre-Réforme Catholique du Concile de Trente.

8 Le Cuestionario de langue française (8-V-1939) est en fait un programme qui fixe, pour la première année, les contenus de "phonologie», de morphologie et de syntaxe. De brèves indications méthodologiques suivent ce programme, du type : "Dans la deuxième année, le travail de classe [el estudio] ne se basera plus sur la mémorisation, mais plutôt sur la systématisation et l'explication ». Un schéma de classe est établi pour la deuxième année de français :

- révision systématique des «thèmes » de la première année ;

- nouveau «thème » de morphologie et de syntaxe ;

- traduction directe (version) et « inverse » (thème) de morceaux en rapport au « thème " grammatical ;

- conversation pratique et étude du vocabulaire correspondant ;

- lecture, traduction et explication d'un morceau de littérature simple (au féminin : c'est la littérature qui est simple, pas le morceau)

-f) récitation de morceaux littéraires simples, principalement des poésies apprises par cœur.

Pour les cours postérieurs, les thèmes grammaticaux sont plus compliqués, les textes littéraires et leur traduction de même ; des synthèses de littérature française (du XVIe au XXe siècle), accompagnées des morceaux choisis correspondants, pourront être exposées par les élèves : en clair, les élèves doivent mémoriser et réciter oralement ou écrire, parfois sans rien comprendre, des abrégés de littérature française composés par les auteurs de manuels. Le marché est inondé ainsi de ces Histoires de la littérature française, qui doivent bien sûr être autorisées avant d'être publiées comme manuels. Si un écrivain possède un caractère prestigieux dans l'histoire littéraire, mais " moralement réprouvable ou à tendances idéologiques ou religieuses erronées, il faudrait que le professeur recommande d'éviter la lecture de ses œuvres et mette en relief ses erreurs et son immoralité. »! 
10 La Ley sobre Ordenación de Enseñanza Media (loi d'orientation de l'enseignement secondaire) de 1953 (BOE 27-11-1953), ainsi que le décret correspondant qui fixe le nouveau Plan d'études (Decreto [...], 12-VI-1953, BOE 2-VII-1953) visent à corriger certains défauts observés dans le Plan d'études de 1938 : par exemple, on recherche la " sélection des meilleurs " avec l'établissement d'un examen à la fin de la quatrième année du Bachillerato ; on réduit certains programmes surchargés ; on fixe un double itinéraire de sciences ou de lettres dans les deux dernières années du Bachillerato. Bien sûr, on garantit les droits de l'État, de l'Église et de la Famille dans l'éducation des jeunes gens ; la " formation de l'esprit national » (destinée à affermir " l'éducation morale » et le « sens de la solidarité et de la fidélité dans l'accomplissement des devoirs et dans le service au destin universel de la patrie», section II, loi du 26-11-1953), ainsi que $1^{\prime}$ '« éducation physique», et, pour les jeunes filles en plus, les enseignements ménagers (educación para el hogar) «seront fondamentaux et obligatoires, et feront l'objet de matières dans tous les cours, dans les examens et dans les épreuves menant aux Diplômes de fin d'études » (article 3, décret du 12 VI-1953). Bien sûr, garçons et filles continuaient d'être scolarisés dans des établissements séparés ; on autorise des établissements mixtes, mais garçons et filles seront strictement séparés.

11 Le Plan d'Études de 1953 était accompagné d'un nouveau Cuestionario pour toutes les matières, sauf, curieusement, pour les langues étrangères (Orden del 21-1-1954, BOE 10-11-1954) : les auteurs de manuels et les professeurs continueront donc de se baser sur ceux établis en 1939.

12 Pendant toute cette période, l'Espagne, repliée sur elle-même, reste à l'écart de l'Europe dans les débats et les recherches qui concernent l'enseignement des langues étrangères. Quelques voix s'élèvent cependant peu à peu, qui montrent que l'intérêt d'aller voir ce qui se passe ailleurs n'avait pas disparu. Ainsi P. Sicart est l'auteur d'un ouvrage (Metodología Científica de las Lenguas Vivas en 1947), très important pour connaître cette période : il y réalise un bref parcours historique, il analyse les méthodes d'enseignement, le rôle des langues dans l'éducation, la formation des professeurs, l'apport des sciences connexes (linguistique, psychologie, philosophie), pour conclure qu'il faut construire une méthodologie scientifique d'enseignement des langues. Cependant, il n'est nullement cité par les revues éducatives de l'époque, même pas par la Revista Nacional de Educación, éditée par le ministère de l'Éducation, pourtant éditeur de l'ouvrage de Sicart ${ }^{2}$.

L. Echevarría, professeur d'anglais, auteur de plusieurs ouvrages (voir références bibliographiques), défend une méthodologie mixte (qu'il nomme método directo inorgánico : on commence par la méthode directe au début, puis on passe à la méthode traditionnelle). Il considère que la langue n'est point constituée par un catalogue " inorganique » de mots, mais par un ensemble organique de phrases. Il considère qu'un élève n'a pas le temps réel d'apprendre tous les mots qui lui sont proposés, et qu'il faudrait réduire le nombre de mots à 600 dans chaque cours (Morales 2003 : 203) ; Urbano propose quant à elle le chiffre de 1500 pour toute la scolarité (Urbano 2003 : 211). L. Echevarria reprend les rapports de la VIe Conférence Internationale de l'Instruction Publique, tenue à Genève en 1937, pour défendre l'importance de l'objectif pratique dans l'enseignement des langues vivantes, objectif qui devrait être prioritaire dans les Écoles de Commerce ou similaires.

Les quelques voix qui se hasardent à dire quelque chose proviennent donc des marges $\mathrm{du}$ domaine éducatif : l'enseignement pour enfants, les écoles de langues ou de 
commerce, où était ressentie moins fortement la stricte surveillance à laquelle était soumis l'enseignement dans les établissements scolaires officiels (lycées, universités, écoles normales).

\section{Les premières mentions du Français élémentaire}

Il faut attendre le début des années 50 pour que certains échos de l'Europe commencent à arriver en Espagne, et que certains professeurs se hasardent à sortir d'Espagne et à assister à des Congrès Internationaux. C'est le cas de G. de Reyna qui publie un rapport détaillé du Congrès pour l'Étude des Méthodes Audio-visuelles dans l'Éducation de Base, organisé à l'Université catholique de Milan, en 1952, sous le patronage de l'UNESCO (voir références). C'est le cas aussi de M. Lago et de Mme Martinez qui participent au Congrès de la Fédération Internationale des Professeurs de Langues Vivantes, tenu à Paris en 1953, et qui y prennent la parole pour donner leurs points de vue.

C'est dans ce contexte d'une légère ouverture que les recherches menées en France sur le Français élémentaire (1954) arrivent en Espagne. Tout d'abord, grâce à l'initiative de certains professeurs : c'est le cas de O. López Fanego (1956), auteure d'une série d'articles sur la question, et de E. Moreu Rey, qui publie un Vocabulario básico francés (1957), qui reprend et traduit le Français élémentaire. Nous reviendrons un peu plus loin sur le rôle de ces professeurs. Mais aussi, grâce aux Instructions ministérielles (Cuestionarios) de 1957. Quelles ont été les répercussions de ces initiatives sur l'enseignement du français? Et plus précisément, sur l'enseignement du vocabulaire ? Voilà ce que nous proposons d'éclairer dans les pages suivantes. Disons d'emblée que l'influence du Français élémentaire va s'étendre assez largement en Espagne, et marquer de manière décisive l'enseignement du FLE, surtout avec la publication du manuel du CREDIF : Voix et images de France.

Les professeurs cités, López Fanego, Moreu et d'autres, vont engager un courant que devront suivre après coup les Instructions officielles. Constatant l'absence de nouveaux Programmes pour les langues vivantes - le Plan d'Études de 1953 ne fut pas accompagné de nouveaux Cuestionarios de langues vivantes, et ceux de 1939 restaient le repère officiel pour les professeurs ! - le conseil de rédaction de la Revista de Educación lance un appel à des collaborations pour unifier les critères que devront suivre les professeurs lors des examens de fin du premier cycle secondaire (examen de Grado) et fin du deuxième cycle (Reválida de Bachillerato) : les nouvelles recherches entreprises sur le Basic English devraient permettre une certaine homogénéisation des niveaux d'exigence. Après un premier numéro dédié à l'anglais ( $n^{\circ} 43$, avril 1956), un deuxième numéro reprend les opinions des professeurs de français ( $n^{\circ} 44$, mai 1956). Ce numéro publie en 1956 un article de López Fanego, agrégée du lycée Miguel de Cervantes de Madrid, qui donnera lieu à des réactions de la part d'autres professeurs de français qui envoient des lettres à la Revue.

Dans cet article, López Fanego résume l'émergence du Français élémentaire (FE), la méthode d'élaboration, les applications déjà faites (le manuel : Le Français élémentaire, méthode progressive de français parlé, par G. Gougenheim et G. Mauger, directeur de l'École pratique de l'Alliance française), mais aussi les critiques dont cette initiative fut l'objet. Elle reprend certaines objections de M. Cohen, de G. Serbat, notamment l'idée que le FE est insuffisant pour exprimer toutes les notions complexes de la civilisation actuelle et de la culture française. Le Français élémentaire est ainsi situé à sa juste place 
: étant donné qu'on ne peut pas tout apprendre à la fois, il faut procéder à une première sélection, et à une gradation rationnelle, question sur laquelle G. Gougenheim se montrait également d'accord.

19 Les réponses d'autres professeurs ne se font pas attendre : M. Grande Ramos, agrégé du lycée de garçons de Bilbao, sans s'opposer frontalement aux idées de Lopez Fanego, répond que le vocabulaire de base n'est autre chose que ce qu'on appelait auparavant " les termes usuels de la langue courante ", et qu'il faudrait, avant de déterminer le vocabulaire de base, décider quelle portée il faut donner aux études de français, etc. Il finit par proposer son propre manuel (Lengua francesa I) comme l'exemple à suivre sur cette question. $\mathrm{O}$. López Fanego répondra à $\mathrm{M}$. Grande, ainsi qu'à d'autres lettres reçues par la rédaction de la Revista de Educación (des professeurs W. Merino, E. Vázquez, H. Giraldo...), dans un nouvel article publié dans ce même numéro. Il faut réformer bien sûr le Plan d'études en vigueur, en augmenter les années ; elle propose de limiter le français élémentaire aux deux premières années d'étude du français, mais il ne s'agit nullement de réduire le vocabulaire à ces quelque 1300 mots, bien au contraire, dit-elle. Un autre article, dans le même numéro, est consacré à la question de la didactique du vocabulaire (rédigé par M. Muñez Cortés, pp. 7-11, où il cite, par exemple, le Précis de sémantique française de S. Ullman, ou l'article de R. Dottrens et D. Massarenti sur le «vocabulaire fondamental du français »). Un numéro postérieur ( $n^{\circ} 49$, octobre 1956) s'occupera de la formation des professeurs de langues vivantes : nous voyons ainsi qu'une revue d'éducation générale consacre quatre numéros aux questions de l'enseignement des langues vivantes de l'époque ! La question du vocabulaire de base s'étend même au latin : le n 46 (juin 1956) publie un article de M. Mathy, professeur de Lettres au lycée d'Annecy, qui avait été l'auteur en 1952 d'un vocabulaire de base du latin (Paris, éd. OCDL).

Quant à Moreu, il compose un ouvrage de 110 pages (Vocabulario Básico Francés, 1957), sur la base du Français élémentaire (éd. de 1954), mais avec plusieurs modifications qui en augmentent l'intérêt pour les professeurs de français espagnols. Tout comme López Fanego, il place le FE sous les auspices de l'UNESCO, moyen, certainement d'éviter toute suspicion de francophilie.

21 Les modifications qu'il introduit sont les suivantes :

22 - Il ajoute, tout au début, les normes principales de la prononciation française, réduites à 5 pages (pp. 5-9), ce qui montre un effort important de synthèse.

23 - Il ajoute, à la fin du catalogue des mots de base, des annexes pour compléter le document de travail : la liste des mots ordonnés alphabétiquement en espagnol, avec la traduction correspondante en français (pp. 77-91) ; le vocabulaire de base classé par ordre « idéologique » (nombres, lieu, forme, poids, temps, mesure, monde, campagne, animaux, corps, vie, sens, sentir, esprit, famille, gens, le bien et le mal, jeu, art, pays, religion, maison, ville, costume, repas, travail, métier, outils, voyages, affaires, la classe : pp. 91-99). Ce classement thématique, par centres d'intérêt, s'insère dans la tradition des catalogues antérieurs, mais possède l'avantage de comparer les réductions proposées et facilite la tâche des rédacteurs éventuels de manuels.

24 - L'ouvrage contient également (annexe 3) un abrégé de grammaire française (Compendio grammatical : pp. 101-109), réduit aux questions suivantes : formation du pluriel et du féminin, les articles, les adjectifs, les pronoms, les verbes, l'interrogation, la préposition et la construction de la phrase, l'accord du participe passé. Par rapport à la grammaire contenue dans le Français élémentaire, il présente les questions de 
grammaire de base dans un ordre traditionnel, pour que les futurs rédacteurs de manuels puissent retrouver facilement la question grammaticale à inclure (ou à exclure).

Quant au catalogue de mots proprement dit, par rapport au Français élémentaire de 1954, Moreu réalise plusieurs modifications :

-Il ajoute et retranche une série de mots (voir les listes dans l'Annexe 1).

-Il propose la traduction en espagnol de tous les mots, dans une colonne parallèle.

-Les mots sont accompagnés de l'article indéfini pour en indiquer le genre, ou bien pour différencier le nom du verbe.

29 -Il met le pluriel de quelques mots quand la formation du pluriel est irrégulière (par exemple : un animal, des animaux).

30 -Il ajoute le féminin de certains adjectifs (adroite, amusante, gentille, masculine, paysanne, etc.).

31 -Pour faciliter la prononciation, il met en italiques certains caractères graphiques de quelques mots qui peuvent présenter des difficultés pour les étudiants espagnols: les consonnes doubles, des mots comme « annoncer, aiguille, alphabet, automne », etc.

-Il met des exemples d'usage, ou bien il introduit le mot en question dans de courtes phrases pour faciliter la mémorisation du mot et augmenter la capacité d'expression (au lieu de " avoir peur », on trouve « elle a peur »).

33 -Il enlève l'indication concernant la partie du discours dont il s'agit (entre parenthèses dans le FE), et la remplace par un exemple : une bête (une grosse bête) ; bête (tu es trop bête !).

-Il conjugue les verbes (par exemple : s'asseoir- je m'assieds (je m'assois), nous nous asseyons, je me suis assis ; employer -j'emploie, nous employons).

-Il introduit de petites remarques en pied de page, sur la prononciation, la traduction du mot en catalan et en portugais, etc.

Quant aux Instructions officielles, disons que le Plan d'études de 1953 sera légèrement modifié en 1957, donnant lieu, cette fois, à de nouveaux Programmes pour les langues vivantes (BOE 21-IV-1958). Parmi les objectifs fixés pour la première année de français ( $2^{\circ}$ curso), le législateur stipule l'acquisition des éléments essentiels de phonologie et de morphologie, la pratique de lectures expliquées, et de façon précise «l'acquisition ferme du Vocabulaire Élémentaire». L'allusion au Français élémentaire, c'est-à-dire à l'ouvrage de Gougenheim, Michéa, Rivenc et Sauvageot, se précise un peu plus loin : « Les vocabulaires, groupés pour leur étude ou apprentissage, comprendront les mots usuels, de préférence ceux qui sont inclus dans le Vocabulaire du Français élémentaire [en français dans le texte].

On pourrait saluer la mention explicite au Français élémentaire dans le Programme officiel comme la démonstration d'une nouvelle sensibilité des responsables de l'éducation espagnole à l'égard de l'enseignement des langues vivantes. Ce serait trop dire. En effet, si le Français élémentaire est recommandé, le vocabulaire à proposer aux élèves ne doit pas être tiré de ce catalogue « de façon exclusive ». La suite du texte législatif montre les limites de ce Cuestionario :

Ces vocabulaires ne seront pas accompagnés de leur traduction ; c'est le professeur qui devra la donner, et les élèves la retiendront par cœur ou la noteront dans leurs cahiers. Les centres d'intérêt seront : la famille, la maison et ses installations, etc. 
Le travail : les vacances, etc. La ville : la poste, le télégraphe, les téléphones, la gare, le train, les bus, la route. Les spectacles : sports, cinéma, cirque, théâtre, etc.

L'art : la musique, la radio, la télévision, la sculpture, la peinture, l'architecture. La navigation maritime et aérienne. Le marché : magasins et produits alimentaires, etc. Les fêtes : populaires et religieuses, etc. (BOE 21-IV-1958: 1055) ${ }^{3}$.

Quant aux procédés pour faire acquérir aux élèves cette " acquisition
vocabulaire élémentaire », ce programme conseille la démarche suivante :

Naturellement, les termes de vocabulaire contenus dans le « Français Élémentaire " [en français dans le texte et avec des guillemets] seront distribués le long de toute l'année, et il faut que les élèves puissent le consulter. Pour cela on recommande que les manuels l'insèrent dans les pages finales avec la traduction espagnole correspondante, en ne consignant que deux acceptions pour chaque mot pour éviter les confusions chez les élèves $(1957: 1056)^{4}$.

Le rédacteur du Programme est au courant des recherches qui se font en France, bien sûr : ce qui est moins sûr est qu'il en ait compris le sens, ou même, qu'il partage les implications d'une telle démarche. En effet, aucune limitation n'est fixée pour la deuxième année ( $3^{\circ}$ curso) : la progression du vocabulaire est éliminée au profit du système des centres d'intérêt ou des « temas » traditionnel (le travail : la ferme, l'usine, l'artisanat ; les sports et les loisirs ; la science et l'art ; l'Église et l'État ; les transports...) (ibid., p. 1063) ; quant à la troisième année de français ( $5^{\circ}$ curso), on passe au " vocabulaire littéraire expliqué » : c'est-à-dire que les élèves doivent extraire des textes choisis les termes d'un usage peu fréquent ou difficiles, les noter dans leurs cahiers avec la traduction ou l'explication correspondante

Les instructions de 1957 ne changent rien: elles ne contraignent personne. Invoquer la liberté de méthode du professeur permet aux «traditionalistes » de rester fermement ancrés sur leurs positions. Parler du système mixte théorico-pratique sans le définir contribue à rassurer le sentiment d'autocomplaisance : tous sont convaincus de pratiquer un tel système, le meilleur de tous. Fixer comme objectif d'apprentissage pour la première année de français le Français élémentaire (1954), qui ne comporte pas moins que 1063 termes, et dire en plus que le vocabulaire fondamental ne doit pas en être tiré " de façon exclusive ", cela signifie ouvrir la porte à n'importe quelle extension, même dans la première année ; extension qui se multiplie dans les cours suivants, où l'on reprend la progression thématique. Tout cela signifie n'avoir rien compris à l'entreprise du Français élémentaire.

41 Ces Instructions ont cependant un point fort : elles permettent à certains de se risquer vers des domaines jusque-là inexplorés (en Espagne) ou même interdits, au point que, au début des années soixante, le renouveau de l'enseignement du FLE en Espagne profite de cette porte légèrement entrouverte, par laquelle va pénétrer un courant d'air de plus en plus fort. Et nous pouvons dire que le fer de lance qui inspire les propositions de renouvellement, c'est précisément le français fondamental, qui rencontre des résistances non moins fortes!

\section{Les répercussions}

Le Centro de Orientation Didáctica, organisme dépendant du ministère de l'Education espagnol, réunit à Madrid en 1961 trente-neuf professeurs agrégés de langues modernes, dans des Journées d'étude consacrées à " analyser les problèmes de l'enseignement des langues vivantes. » Ces trente-neuf agrégés discutent des problèmes 
pendant toute une semaine, d'abord en sous-commissions, puis ils rapportent en réunion plénière leurs conclusions, qui sont adressées au Ministère, et publiées dans le $\mathrm{n}^{\circ} 261$ de la très officielle Revista de Enseñanza Media (1962). Il s'agit donc d'un document exceptionnel pour connaître la situation de l'enseignement des langues vivantes à l'époque.

43 López Fanego, dont nous avons déjà parlé, participe à cette réunion et, en tant que présidente de la commission sur le vocabulaire, expose les conclusions du travail (qui donneront lieu à l'article intitulé " Vocabularios racionales ", voir bibliographie). Elle défend à nouveau de façon énergique la valeur du Français fondamental (expression utilisée depuis 1959), et son intérêt pour l'enseignement de la langue française. On voit qu'elle a dû pactiser dans les conclusions de son article, puisqu'elle est censée reprendre les décisions de la commission et de la séance plénière. Elle défend l'idée qu'on doit concéder au vocabulaire une importance égale à la grammaire, que ce vocabulaire doit être composé de façon rationnelle et graduée, sur la base du Français fondamental, mais en l'adaptant aux besoins des réalités de l'enseignement espagnol. Dans sa bibliographie, elle cite les ouvrages suivants, entre autres : Vocabulaire de base et formation intellectuelle, de R. Michéa (Anvers : 1949) ; Vocabulaire fondamental du français, de R. Dottrens et D. Massarenti (Genève, Institut J.J. Rousseau), et Le vocabulaire fondamental du français, de J. D. Hay good (Genève). L'Espagne était ouverte à l'extérieur, définitivement.

La suite est mieux connue. L. Grandia Riba, inspecteur de l'enseignement secondaire, fait la traduction du Français fondamental, premier et deuxième degré (1962). C'est le ministère de l'Éducation nationale qui lui a commandité la traduction de cet ouvrage. L'auteur fait une présentation du français fondamental, explique en quoi il consiste, comment il a été conçu. À la fin du prologue, il explique qu'il a fait la traduction des mots en espagnol pour en faciliter la consultation; il a également ajouté un index général de tous les mots qui sont dans le FF. Le vocabulaire du premier degré va servir pour l'examen de "Grado Elemental », qui se faisait sans dictionnaire et qui consistait en une traduction (version) de morceaux choisis. Son fils, L. Grandia Mateu, professeur de français au lycée Ganivet à Grenada, va développer les idées du FF avec la confection d'une série de manuels d'inspiration audiovisuelle, en créant même un centre de recherche (Centre de Linguistique Appliquée, CELA) ${ }^{5}$.

La Loi sur l'Unification du premier cycle de l'enseignement secondaire de 1967 (BOE 11IV-1967), ainsi que les Cuestionarios pour les langues vivantes correspondantes (BOE 30-IV-1967) reprendront les changements qui s'étaient produits au sein de la communauté des professeurs et que ceux-ci réclamaient. Ces Instructions n'ont plus pour fonction d'indiquer un chemin, mais de consigner dans un texte légal une réalité nouvelle. Dans le cas du français, on limite les mots à assimiler à 400 pour la première année [ $1^{\circ}$ curso] (fixés à partir des centres d'intérêt proches de l'élève : la salle de classe, les mouvements, les couleurs, les formes, le temps, la journée scolaire, les repas, les vêtements, la maison, la famille); à 500 de plus pour la deuxième année [ $2^{\circ}$ curso] (à partir des domaines suivants : la campagne, les animaux et les plantes, la montagne et la mer, les voyages, les transports, la ville, les loisirs) ; à 600 autres pour la troisième année [ $3^{\circ}$ curso] (à partir de nouveaux centres d'intérêt : les magasins, les cafés, les spectacles, les sports, la vie religieuse, les vacances); la quatrième année est destinée à une révision d'ensemble. Les instructions méthodologiques font une référence explicite aux travaux du CREDIF : « même avec des réserves, nous croyons que le premier degré 
[du Vocabulaire fondamental] doit constituer la base des trois premières années de français, avec les additions ou suppressions qu'on pourra considérer comme nécessaires $»^{6}$. Le vocabulaire à assimiler au cours des ces quatre années de français du premier cycle de l'enseignement secondaire ne dépasse pas les 1500 mots, changement important. Par ailleurs, ces instructions méthodologiques comportent un regard vers le futur, dans des formulations du type : auparavant, l'étude d'une langue vivante se faisait dans l'objectif de comprendre un texte écrit, de nos jours, on l'apprend dans le but de l'utiliser, de la parler ; donc la prononciation, le rythme, l'intonation, la musicalité de la langue possèdent une importance extraordinaire ; le professeur devra ainsi capter et reproduire les divers éléments phonologiques du français et les proposer à l'acquisition des élèves. C'est une musique que l'on entendait à l'époque dans d'autres pays : l'Espagne participe à nouveau à ce qui se faisait à la même époque en Europe.

Pour preuve, s'il en fallait une : P. Rivenc et A. Rojo Sastre effectueront une enquête de fréquence qui permettra d'établir l'espagnol fondamental (ainsi qu'un portugais fondamental), en perfectionnant la méthode de travail qui avait été utilisée pour le français : " en donnant plus d'ampleur au corpus recueilli (Fr : 312133 occurrences ; Esp : 800000 occurrences [...]), en augmentant considérablement le nombre des informateurs ( $\mathrm{Fr}: 275$; Esp. 1600 ), et surtout en constituant ces corpus sur des bases beaucoup plus rigoureuses ; l'automatisation des échantillons de populations tenant compte de données socioculturelles précises » (Rivenc $1979: 17$, note).

\section{BIBLIOGRAPHIE}

BESSE, H. (1979), « Contribution à l'histoire du Français fondamental », Le Français dans le Monde 148, pp. 23-30.

COHEN, M. et al. (1955), Français élémentaire, non! Paris : Éditions Sociales.

ECHEVARRÍA, L. (1950), « La enseñanza del inglés a los niños », Bordón 12, pp. 37-39.

ECHEVARRÍA, L. (1952), «La enseñanza del inglés por el Método Orgánico Directo », Revista española de Pedagogía, año 37, pp. 143-147.

GOUGENHEIM, G. (1956), «Français élémentaire, oui ou non?», Bulletin de la Faculté des Lettres de Strasbourg, pp. 8-9.

GOUGENHEIM, G. (1958), Dictionnaire fondamental, Paris : Didier

GOUGENHEIM, G., MICHEA, P., RIVENC, P. et SAUVAGEOT, A. (1956), L'élaboration du Français élémentaire, Paris : Didier.

GRANDIA RIBA, L. (1962), El Francés fundamental, Madrid : Dirección General de Enseñanza Media. LOPEZ BARRERA, J. (1927), Vocabulario usual de la lengua francesa, Santander : Aldus.

LÓPEZ FANEGO, O. (1956), «La selección del vocabulario en la Enseñanza del Francés », Revista de Educación 44, pp. 68-71. 
LÓPEZ FANEGO, O. (1962), «Vocabularios racionales », Revista de Enseñanza Media 99-102, pp. 457-471.

MAUGER, G. et GOUGENHEIM, G. (1961), Instructions pédagogiques pour l'emploi du Français élémentaire, Paris : Hachette.

MORALES Gil, J. (2003), La evolución metodológica de la enseñanza oficial del francés en España : 1936-1970, 2 vol., Universidad de Huelva, thèse non publiée.

MOREU REY, E. (1957), Vocabulario Básico Francés, Barcelona : Teide.

REYNA, G. (1952), «Los métodos audio-visuales en la educación fundamental », Revista de Educación 2, pp. 164-167.

RIVENC, P. s.a., «Deux méthodes audiovisuelles d'enseignement du français comme langue étrangère ", Paris : CRÉDIF, brochure ronéotée.

RlVENC, P. (1979), « Le Français fondamental vingt-cinq ans après », Le Français dans le Monde 148, pp. 15-22.

SICART, P. (1947), Metodología Científica de las Lenguas Vivas, Madrid : MEN.

URBANO MARCHI, B. (2003), La enseñanza-aprendizaje del francés como lengua extranjera en España durante la época franquista (1938-1970), Universidad de Granada, thèse non publiée.

Documents officiels

Cuestionario de Francés, Ministerio de Instruction Pública y Bellas Artes, Gaceta de Madrid, 21 de Octubre de 1934.

Le Français élémentaire (1955), Publication du Centre National de Documentation Pédagogique.

Le Français élémentaire (1957), Cahiers Pédagogiques pour l'enseignement du second degré 7 : « Efficacité et limitation dans l'enseignement », de G. Gougenheim (p. 45), « Français Élémentaire et Pédagogie», de P. Ginestier (p. 47), «Une expérience d'enseignement du Français par les méthodes audio-visuelles en Yougoslavie », de P. Rivenc (p. 50).

Ministère de l'Éducation Nationale (1964), Le Français fondamental (1er degré: 1300 mots - 2ème degré : 1000 mots, Paris, Institut Pédagogique National. (3e éd. 1973), ministère de l'Éducation nationale, École Normale Supérieure de Saint-Cloud, et CREDIF.

Ministère de l'Éducation nationale (1966), Le Français fondamental. 1er degré. Précédemment " Français élémentaire», Paris : Institut Pédagogique National

\section{ANNEXES}

Mots ajoutés au Français élémentaire (1954) par E. Moreu (1957)

accent - addition - additionner - alphabet - angle - annoncer -ascenseur - aussi... que autant... que - avant-hier car (un car) - centimètre - clé - citron - coiffeur - col (de chemise) -cahier - costume - course - couture - couturière - couvert - craie - cri cuillère, défense

encre - église - espagnol - étonnant

il faut (au lieu de falloir) - fauteuil - féminin

gomme - gramme 
imperméable

jusqu'à

malle - masculin - masculine - métro

nationalité

omelette - orange

page - parapluie - passeport - patrie - poêle - porteur - poudre - potage -prénom -

présent - proche - professeur - pyjama règle - remercier - retard - retour - robinet salon - salle à manger - sein - soin - stylo

tante - toilette - tour

visiter - voici

Soit presque 70 mots nouveaux dont un certain nombre concerne la salle de classe.

Mots retranchés au Français élémentaire (1954) par E. Moreu (1957)

blouse

ceinture - cuiller - cultivateur

débrouiller - Dieu (avec des majuscules; il garde : dieu)

foin - forge - forger - forgeron - France

milliard

parente (féminin de : parent, qui figure au FE) - phono - pharmacien -

pioche - piocher - pomme

thé

Soit 18 mots enlevés

\section{NOTES}

1. Traduit par moi.

2. Il n'existe, à l'époque, qu'une seule référence à l'ouvrage de Sicart, dans Lingüística española y metodología de la enseñanza del idioma, de Ginés Garcia Martinez ([1951] 1954, Cartagena).

3. Traduit par moi.

4. Traduit par moi.

5. Nous conseillons à ceux qui sont intéressés par ce sujet de se reporter à l'article : « Le centre expérimental de linguistique appliqué de Granada. Vingt ans d'histoire dans le renouvellement des méthodologies des LVE en Espagne ", que nous avons présenté au Colloque international pour le centenaire des étudiants étrangers ", célébré en 1996, et qui a été publié dans les Actes de ce colloque ( $\mathrm{L}$ 'apport des centres de français langue étrangère à la didactique des langues, $\mathrm{n}^{\circ}$ dirigé par D. Abry et R. Bouchard, Grenoble, 1999).

6. Traduit par moi. 


\section{AUTEUR}

MARIA EUGENIA FERNÁNDEZ FRAILE

Université de Grenade 